\title{
UNSWIRF: the University of New South Wales Infrared Fabry-Perot
}

\author{
Lori E. Allen ${ }^{a}$, Michael C. B. Ashley ${ }^{a}$, Michael G. Burton ${ }^{a}$, \\ Stuart D. Ryder ${ }^{b}$, John W. Storey ${ }^{a}$, and Yin-Sheng Sun ${ }^{a}$ \\ ${ }^{a}$ Department of Astrophysics and Optics, School of Physics \\ University of New South Wales, Sydney 2052, Australia \\ ${ }^{b}$ Joint Astronomy Centre, 660 N. A'Ohoku Place \\ Hilo, HI 96720, USA
}

\begin{abstract}
We describe an imaging Fabry-Perot instrument, and give examples of its astronomical applications. The salient features of the instrument are: wide wavelength coverage $(1.5$ to $2.4 \mu \mathrm{m})$ with a single etalon, operation near the focal plane in a converging beam, resolving power $\mathrm{R} \sim 4000$, relatively easy portability to other telescopes.
\end{abstract}

Keywords: Fabry-Perot etalon, near-infrared

\section{INTRODUCTION}

The UNSW Infrared Fabry-Perot (UNSWIRF) is a narrow-band (R 4000) tunable filter for the near-infrared (1.5 to $2.4 \mu \mathrm{m}$ ), designed to work with array cameras in an $\mathrm{f} / 18$ (or slower) beam, with a diameter of $<70 \mathrm{~mm}$. Currently, UNSWIRF is used in conjunction with the IRIS camera at the $\mathrm{f} / 36$ focus of the Anglo-Australian Telescope (AAT). The etalon is a model ET70WF from Queensgate Instruments Ltd, with unique coatings that are able to operate all the way from 1.5 to $2.4 \mu \mathrm{m}$. Potential applications of the instrument range from the study of nearby star forming regions, ${ }^{1,2}$ planetary nebulae ${ }^{3}$ and supernova remnants, to the nuclei and disks of nearby and active galaxies. ${ }^{4}$

\section{INSTRUMENT DESCRIPTION AND PERFORMANCE}

A detailed description of UNSWIRF can be found in Ref. 5. A schematic of the instrument is shown in Figure 1. The etalon rests in a high-precision slide, mounted between IRIS (a near-infrared camera available on the AAT) and the Cassegrain focus. The slide drives UNSWIRF in and out of the beam as required, with sufficient precision $(\sim 10 \mu \mathrm{m})$ that it is not necessary to repeat flat-field exposures. An interesting feature of the instrument is that the etalon is placed close to the focal plane, in a slowly converging beam, rather than using collimated light. The $\mathrm{f} / 36$ beam is sufficiently slow that the resolution of the etalon is unaffected. Being close to the focal plane is advantageous for correcting localized plate defects (e.g., small variations in the plate spacing) that would reduce the overall finesse if in a collimated beam.

Communication with UNSWIRF (e.g., to change the etalon spacing) is via a PC interface ${ }^{6}$ to a Queensgate Instrument Ltd CS100 controller, which rides in the Cassegrain cage. All UNSWIRF control commands can be issued from the AAT control room, within simple "run" files which also control telescope offsets and detector functions. IRIS camera optics provide two imaging modes: the wide field is a $1.7^{\prime}$ circular field of view with a pixel scale of $0.77^{\prime \prime} /$ pixel; the intermediate field optics provide a $34^{\prime \prime} \times 34^{\prime \prime}$ field of view with $0.27^{\prime \prime} /$ pixel.

Reduction and analysis of UNSWIRF images is aided by a suite of IRAF-based scripts. These scripts were written to perform all the basic steps of data reduction, including cleaning, flat-fielding, sky and continuum subtraction, cube building, line profile fitting, photometry of standard stars and flux calibration. They are available* as an IRAF add-on package to all users of UNSWIRF, and may be useful for the analysis of similar data from other instruments.

Further author information -

M.C.B.A (correspondence): Email: mcba@newt.phys.unsw.edu.au

${ }^{*}$ UNSWIRF WWW site: http://www.phys.unsw.edu.au/ sdr/unswirf/UNSWIRF.html 
Table 1. Measured UNSWIRF Performance

\begin{tabular}{cccccc}
\hline $\begin{array}{c}\text { Wavelength } \\
(\mu \mathrm{m})\end{array}$ & $\begin{array}{c}\text { FWHM } \\
(\AA)\end{array}$ & $\begin{array}{c}\text { Resolving } \\
\text { Power }\end{array}$ & Finesse & $\begin{array}{c}\text { Equivalent } \\
\text { Width }(\AA)\end{array}$ & Sensitivity $^{a}$ \\
\hline 1.644 & 4.1 & 4012 & 63 & 6.5 & 2.4 \\
1.652 & 3.6 & 4584 & 72 & 5.6 & $\ldots$ \\
2.117 & 4.6 & 4568 & 91 & 7.2 & 1.2 \\
2.153 & 6.2 & 3492 & 71 & 9.7 & 1.7 \\
2.190 & 5.4 & 4047 & 84 & 8.5 & $\ldots$ \\
2.249 & 7.0 & 3191 & 68 & 11.0 & $\ldots$ \\
2.334 & 6.7 & 3492 & 78 & 10.4 & $\ldots$ \\
\hline
\end{tabular}

${ }^{a} 5-\sigma$ detection in a single 120 s exposure near the quoted wavelength, in units of $10^{-15} \mathrm{erg} \mathrm{cm}^{-2} \mathrm{~s}^{-1} \operatorname{arcsec}^{-2}$, after sky subtraction and flat-fielding.

\section{USING UNSWIRF TO INVESTIGATE PHOTODISSOCIATION REGIONS}

Photodissociation regions (PDRs) are regions where stellar energy is deposited onto the surface layers $\left(A_{v}<10\right)$ of molecular clouds, and are generally found in sites of recent star formation and in planetary nebulae. Most previous studies of near-infrared line emission from PDRs have suffered from either poor spectral resolution, poor spatial resolution, or poor areal coverage. UNSWIRF solves these problems, thus we have initiated a program to investigate the internal structure (e.g., variations in gas temperature and density) of Galactic PDRs. A few examples are shown here.

\subsection{NGC 3132}

Images of the planetary nebula NGC 3132 were made in the $\mathrm{H}_{2} 1-0$ and $2-1 \mathrm{~S}(1)$ lines at $2.12 \mu \mathrm{m}$ and $2.25 \mu \mathrm{m}$, respectively, and in the $\mathrm{HI} \mathrm{Br} \gamma$ line at $2.16 \mu \mathrm{m}$. The $1-0$ and $\mathrm{Br} \gamma$ images are shown in Figure 2, in which a central ionized cavity is clearly confined by shells of $\mathrm{H}_{2}$.

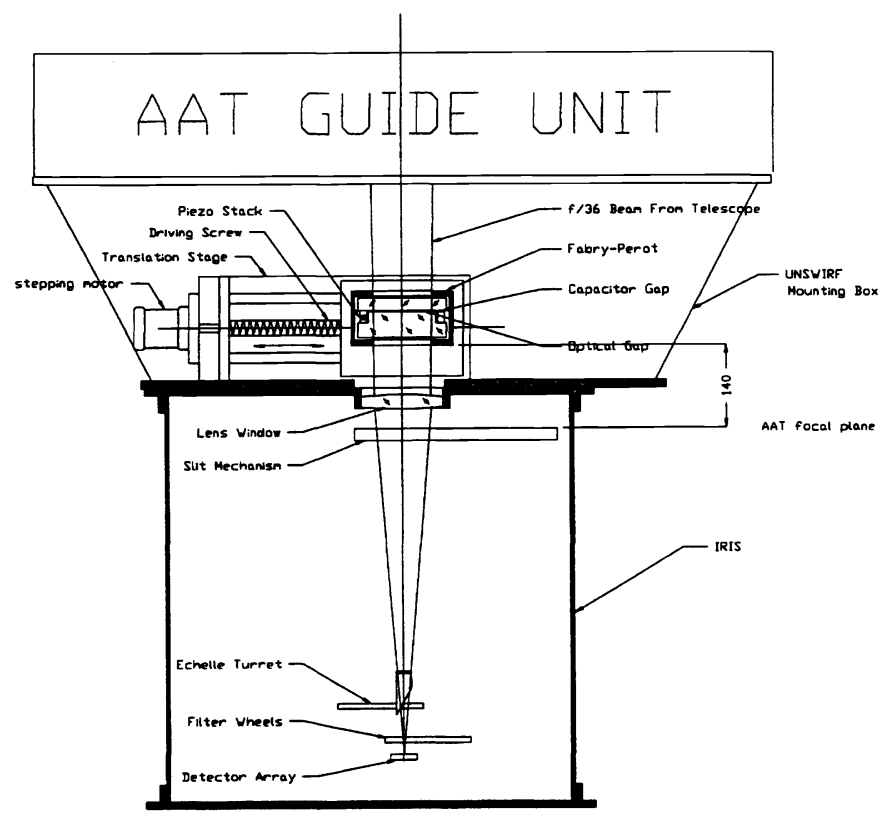

Figure 1. Schematic of UNSWIRF, showing the instrument in its mounting box between IRIS and the Cassegrain focus of the AAT. 
Table 2. Blocking Filters

\begin{tabular}{cccl}
\hline $\begin{array}{c}\text { Central wavelength } \\
(\mu \mathrm{m})\end{array}$ & $\begin{array}{c}\text { FWHM } \\
(\mathrm{nm})\end{array}$ & $\begin{array}{c}\text { Filter } \\
\text { Wheel }\end{array}$ & Nominal use \\
\hline 1.64 & 16 & Upper & {$[$ Fe II] $($ Galactic $)$} \\
1.65 & 15 & Upper & {$[\mathrm{Fe} \mathrm{II}](0.002<\mathrm{z}<0.006)$} \\
1.74 &.. & $\ldots$. & $\mathrm{He} \mathrm{I}$ \\
2.12 & 26 & Lower & $\mathrm{H}_{2} 1-0 \mathrm{~S}(1)$ \\
2.16 & 32 & Upper & $\mathrm{HI} \mathrm{Br} \gamma$ \\
2.25 & 24 & Upper & $\mathrm{H}_{2} 2-1 \mathrm{~S}(1)$ \\
2.21 & 93 & Lower & Continuum \\
2.34 & 86 & Lower & CO/continum \\
\hline
\end{tabular}

${ }^{a}$ As of March 1998. Check the UNSWIRF Web page for current configurations at http://www.phys.unsw.edu.au/ sdr/unswirf/UNSWIRF.html

Seen plane-by-plane (Figure 3), the $\mathrm{H}_{2}$ (1-0) data cube suggests a complicated kinematic structure. The cube was run through a Lorentzian fitting routine to produce an intensity-weighted velocity map, with a resolution of about $1 \mathrm{~km} / \mathrm{s}$. The map shows that the total range in velocity within NGC 3132 is $\sim 50 \mathrm{~km} / \mathrm{s}$.

\subsection{Carina}

A quick-look survey of the Keyhole Nebula neighborhood in Carina turned up several $\mathrm{H}_{2}$ emitting regions, including the one in Figure 4. The interesting morphology of this region led us to dub it the "Kangaroo nebula".

\subsection{The Eagle Nebula}

The "elephant trunk" features (Figure 5) in the star-forming region M16 (the Eagle Nebula) were imaged in the 1-0 S(1) line of $\mathrm{H}_{2}$, the 2-1 $\mathrm{S}(1)$ line of $\mathrm{H}_{2}$ and the $\mathrm{HI} \mathrm{Br} \gamma$ line. The maps were used to determine how far the UV photons from the nearby $\mathrm{OB}$ association ( $\mathrm{NGC} 6611$ ) penetrate the columns, how dense the gas is, and the mechanism by which the $\mathrm{H}_{2}$ is excited.

$$
\mathrm{H}_{2}:-\mathrm{O}(1) \quad \text { H. } 3 \mathrm{ry}
$$
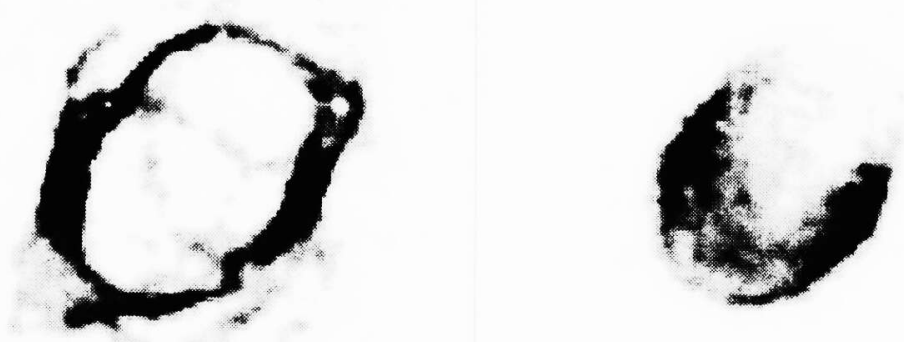

Figure 2. NGC 3132 shows evidence for multiple shells in $\mathrm{H}_{2}$ 1-0 S(1) (left), the innermost of which is filled with $\mathrm{Br}$ ? emission (right). 

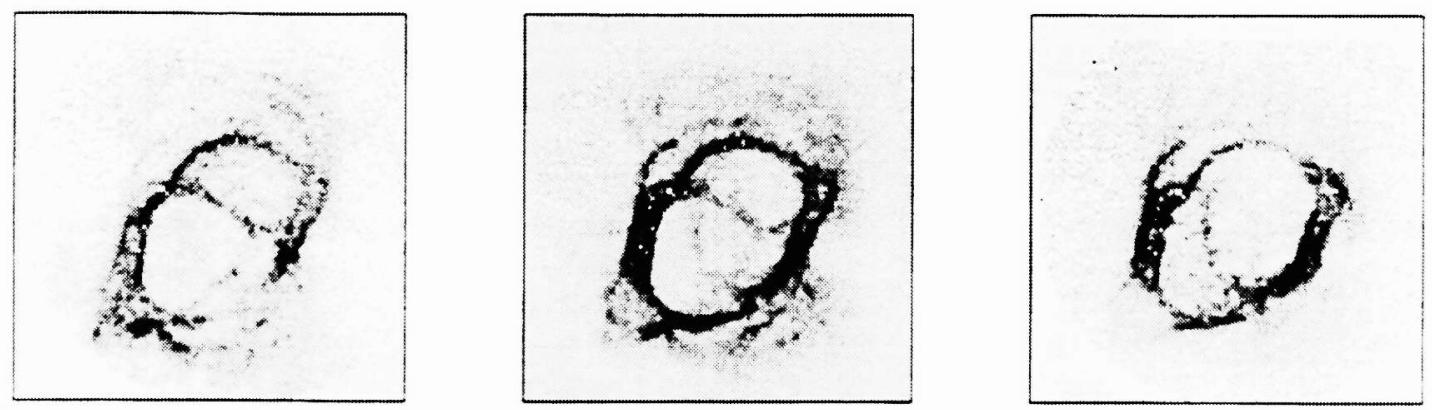

Figure 3. Stepping through the $\mathrm{H}_{2}$ (1-0) line, at intervals of $40 \mathrm{~km} / \mathrm{s}$, with a bandwidth of $60 \mathrm{~km} / \mathrm{s}$ at each step. The $\mathrm{H}_{2}$ emission exists over a wide range of velocity.

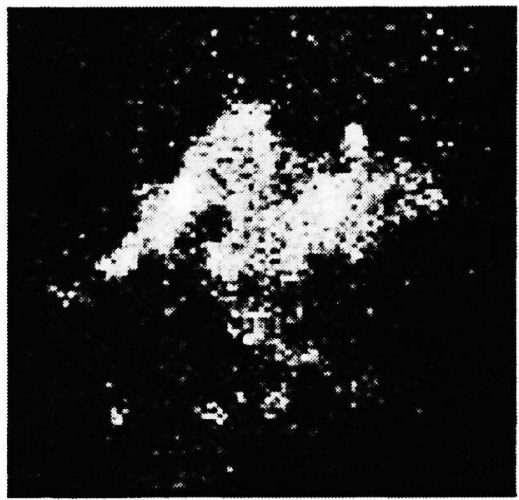

Figure 4. $\mathrm{H}_{2}$ 1-0 S(1) emission from part of the Carina molecular cloud.

\section{SUMMARY}

UNSWIRF proves to be a valuable instrument for PDR studies. It is capable of making high spectral and spatial resolution images that allow us to determine physical conditions inside star-forming molecular clouds.
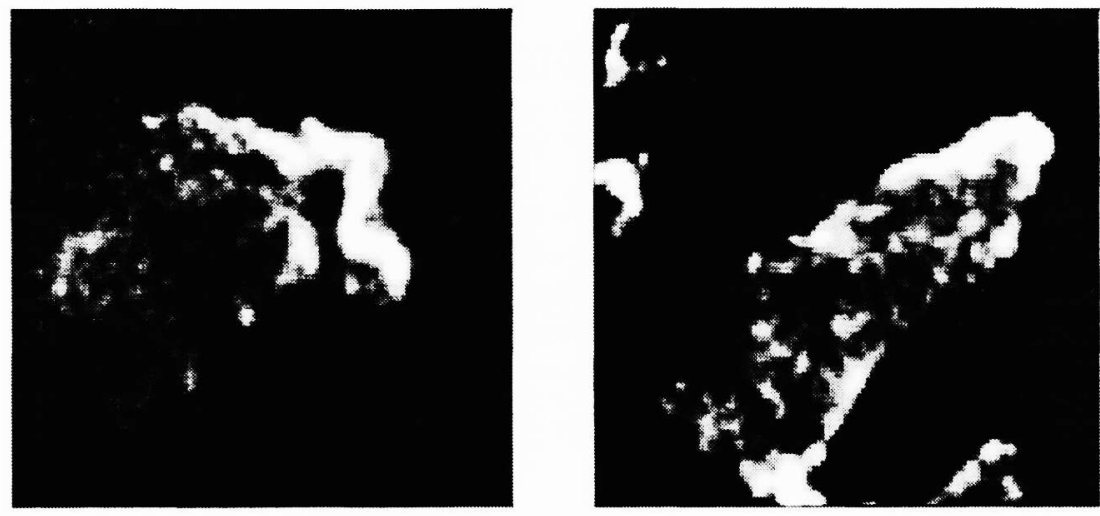

Figure 5. $\mathrm{H}_{2}$ 1-0 S(1) emission from the "elephant trunk" features in M16. 


\section{ACKNOWLEDGMENTS}

UNSWIRF was funded by a grant from the Australian Research Council.

\section{REFERENCES}

1. S. D. Ryder, L. E. Allen, M. G. Burton, M. C. B. Ashley, and J. W. V. Storey, "Molecular Hydrogen Line Emission from the Reflection Nebula Parsamyan 18", MNRAS, 294, 338-346, 1998.

2. L. E. Allen, M. G. Burton, S. D. Ryder, M. C. B. Ashley, and J. W. V. Storey, "Fluorescent Molecular Hydrogen in the Eagle Nebula", MNRAS, submitted, 1998.

3. L. E. Allen, M. C. B. Ashley, S. D. Ryder, J. W. V. Storey, Y-S Sun, M. G. Burton, "IR Fabry-Perot Imaging of NGC 3132 ", in "Planetary Nebulae", eds. H. J. Habing and H. J. G. L. M. Lamers, Proceedings of IAU Symposium 180, Groningen, 1998.

4. R. I Davis, D. A. Forbes, S. D. Ryder, M. C. B. Ashley, M. G. Burton, J. W. V. Storey, L. E. Allen, M. J. Ward, and R. P. Norris, "Near-infrared line and radio continuum imaging of the Circinus galaxy" MNRAS, 293, 189-196, 1998.

5. S. D. Ryder, Y-S Sun, M. C. B. Ashley, M. G. Burton, L. E. Allen, and J. W. V. Storey, “UNSWIRF: A Tunable Imaging Spectrometer for the Near-infrared", Proc. Astron. Soc. Aust., submitted, 1998.

6. M. C. B. Ashley, P. W. Brooks and J. P. Lloyd, "Remote control of astronomical instruments via the Internet," Publ. Astron. Soc. Aust., 13, 17, 1995. 